\title{
ESTIMATIVAS DE CUSTO DA HEPATITE CRÔNICA B NO SISTEMA ÚNICO DE SAÚDE BRASILEIRO EM 2005
}

\author{
adauto Castelo*, Mario G. Pessóa, tânia C.B.B. Barreto, Marcia R.D. Alves, Denizar Vianna araújo \\ Trabalho realizado pelo Centro Paulista de Economia da Saúde - Universidade Federal de São Paulo, São Paulo, SP
}

\begin{abstract}
RESUMO
OBjetivo. O objetivo deste estudo foi estimar o padrão de tratamento, a utilização de recursos e os gastos para cada estágio da HCVB, no ambiente do Sistema Único de Saúde (SUS) do Brasil, no ano 2005.

Métodos. Foi desenvolvido painel Delphi de especialistas para obter informação sobre o padrão de tratamento da HCVB no Brasil. Os dados foram coletados com dez médicos especialistas em hepatologia e doenças infecciosas. A valoração dos recursos foi obtida predominantemente das tabelas de pagamentos do Sistema Único de Saúde e tabelas de preços de medicamentos. As estimativas de custo tiveram a perspectiva do pagador público. Os dados foram analisados estatisticamente pelo programa SPSS 12.0 para Windows.
\end{abstract}

Resultados. Os gastos estimados dos pacientes foram separados em cada estágio da HCVB. O gasto estimado anual por paciente foi: $R$ \$ 980,89 para hepatite $B$ crônica, sem cirrose e sem tratamento antiviral; $R$ \$ 1.243, 17 para cirrose compensada sem tratamento antiviral; $R \$ 22.022,61$ para cirrose descompensada; $R \$ 4.764,95$ para o carcinoma hepatocelular; e $R \$$ 87.372,60 para o transplante hepático.

\section{*Correspondência}

Universidade Federal

de São Paulo

Rua Botucatu, 685 - Vila

Clementino - São Paulo - SP

Cep 04023-062

Tel/Fax: (11) 5549-0158
Conclusäo. Os gastos estimados com procedimentos e medicamentos, excluindo antivirais, representaram os principais componentes do gasto da HCVB. Neste modelo, os gastos aumentam dramaticamente nos estágios mais avançados, sugerindo que retardar a progressão da doença poderá reduzir o gasto no longo prazo.

Unitermos: Hepatite B Crônica. Custos e análise de custo. Custos de Cuidados de saúde.

\section{INTRODUÇÃO}

A infecção crônica pelo vírus da hepatite $B(H C V B)$ é uma grande causa de morbidade e mortalidade no mundo. A estimativa é que 400 milhões de indivíduos estejam infectados e até um milhão morram anualmente pela doença'.

O impacto econômico associado à HCVB é relevante, pois, em áreas endêmicas, o desenvolvimento do carcinoma hepatocelular (CHC) e da cirrose ocorrem na população jovem, acarretando décadas de vida produtiva comprometida.

Inquérito sorológico realizado no Brasil (Rio de Janeiro, Manaus, Porto Alegre e Fortaleza) revelou soroprevalência de antiVHB de 7,9\%, variando de 1,2\% em Fortaleza a 21\% em Manaus². A prevalência do AgHBs inferior a $2 \%$ é considerada baixa, entre $2 \%$ e $8 \%$, intermediária, e alta quando superior a $8 \%$. No Brasil, há áreas representativas dos três níveis de prevalência.

A região amazônica é hiperendêmica em sua porção ocidental, e também a região com maior número de casos de associação de HCVB com o agente delta ${ }^{3}$.

Estudo realizado por Focaccia et al., com método de inferência estatística, utilizando amostra randomizada e estratificada por nível socioeconômico, idade, sexo e local de moradia, realizado na cidade de São Paulo, revelou prevalência de 5,94\% de AgHBs ${ }^{4}$. Vargem Alta, no Espírito Santo, próximo a Cachoeiro do Itapemirim, tem alta prevalência, com soropositividade para 0 AgHBs de $9 \%$, representando outro bolsão de alta endemicidade. Também na região do Vale do Jequitinhonha, em Minas Gerais, foi detectada prevalência de 8,5\%. O Centro-Oeste e o Nordeste consolidaram-se como regiões de baixa prevalência.

O estudo de estimativa de carga de doença constitui um método econômico descritivo que, associado aos dados de prevalência, incidência, morbidade e mortalidade, auxilia na mensuração do impacto para a sociedade decorrente de uma doença específica. Este tipo de estudo varia de acordo com a perspectiva utilizada e os diferentes tipos de custo considerados.

A perspectiva na avaliação dos custos pode ser da sociedade como um todo, somente do agente provedor de saúde, ou do paciente. A escolha da perspectiva terá óbvio impacto nos tipos de custos a serem considerados, com claras implicações metodológicas.

Os custos específicos são identificados e calculados para o estudo do custo da doença. É importante diferenciar "custo" de "preço", assim como as diferentes categorias. Custo é definido como os recursos físicos e humanos consumidos na provisão de um serviço. Preço é estabelecido pelo mercado, inclui margem de lucro e não reflete o custo real para o fornecimento do serviço. Custo direto, uma das categorias de custo, é definido como os recursos físicos e humanos consumidos diretamente na provisão da assistência ao paciente.

Não há estudos nacionais para dimensionamento do impacto socioeconômico da Hepatite B e de suas alternativas de tratamento antiviral. Os cinco medicamentos registrados no Brasil pela ANVISA para tratamento da HCVB são Interferon alfa-2a e $2 b$, Interferon 
Peguilado alfa-2a, Lamivudina, Adefovir e Entecavir ${ }^{5}$.

Em decorrência da escassez de estudos econômicos referentes ao tratamento da HCVB no Brasil, o objetivo do presente estudo foi descrever as estimativas de gasto com o tratamento ambulatorial e hospitalar da HCVB no Sistema Único de Saúde.

\section{Métodos}

Foi realizado painel Delphi com dez especialistas brasileiros nas áreas de hepatologia, infectologia e farmacoeconomia para estimar a conduta local no tratamento da HCVB e de suas complicações. Especialistas participantes representaram os seguintes centros de pesquisa e acompanhamento de pacientes com infecção crônica pelo vírus B no país: Hospital das Clínicas, Instituto do Coração e Instituto de Medicina Tropical da Faculdade de Medicina da Universidade de São Paulo (USP); Universidade Federal de São Paulo; Hospital de Clínicas da Faculdade de Medicina da Universidade Federal de Porto Alegre; Instituto de Infectologia Emílio Ribas, São Paulo; Universidade Federal da Bahia; Hospital do Servidor Público Estadual de São Paulo; Universidade Estadual de Campinas, São Paulo; Universidade Federal do Rio de Janeiro; Centro Paulista de Economia da Saúde e Disciplina de Infectologia, Universidade Federal de São Paulo e Faculdade de Saúde Pública, USP, São Paulo. O questionário, validado em pré-teste com um hepatologista e um infectologista, foi dividido em quatro estágios clínicos associados à HCVB: hepatite crônica sem cirrose, cirrose compensada, cirrose descompensada e carcinoma hepatocelular. Os dados solicitados no questionário foram: uso de terapia antiviral para HCVB, uso de outros medicamentos para o tratamento das complicações relacionadas à HCVB, consultas médicas, testes laboratoriais para monitoramento da progressão da doença, procedimentos e admissões hospitalares, no período de um ano, para estágios específicos de evolução da doença. Os especialistas foram orientados a estimar somente os recursos diagnósticos e terapêuticos para HCVB, desconsiderando outras comorbidades, tais como co-infecção por HIV ou infecção pelo vírus da Hepatite C (HCV).

Com relação ao tempo médio de tratamento com Lamivudina, considerou-se esta medicação administrada por 365 dias, independente da soroconversão.

Somente os gastos resultantes de intervenções diagnósticas e terapêuticas, internação hospitalar, exames complementares, medicamentos e honorários profissionais foram considerados. O impacto econômico da HCVB foi estimado sob o ponto de vista do Sistema Único de Saúde (SUS), no horizonte de tempo de um ano. Aos recursos indicados pelo painel de especialistas foram atribuídos valores em reais, ano de 2005.

Os procedimentos médicos, transplante hepático e insumos utilizados no SUS foram valorados pelos preços reembolsados à rede hospitalar prestadora da assistência médica, por meio da $\mathrm{AlH}$ (Autorização de Internação Hospitalar), disponível no website do DATASUS ${ }^{6}$. Os preços dos medicamentos foram calculados pela publicação "Revista ABC Farma", de maio de 2005. Os valores dos exames complementares também foram obtidos no website do DATASUS. Os dados foram compilados em planilha Excel e analisados estatisticamente pelo programa SPSS 12.0 para Windows.

\section{Resultados \\ Hepatite crônica sem cirrose (HC)}

Nos casos de HC, o tratamento antiviral é recomendado em 39\% dos casos; Lamivudina é o tratamento predominantemente utilizado pelos médicos participantes deste painel de especialistas, representando 59,7\% da preferência, seguida pelo Interferon e Adefovir, com 12,3\% para cada um.

Vale ressaltar que, apesar de indicado em 12,3\% dos casos, o Interferon alfa-2B não tem indicação para o tratamento da Hepatite B Crônica, sendo o seu uso não recomendado em bula.

O gasto médio anual por paciente foi estimado em $\mathrm{R} \$ 10.921,6$, considerando as probabilidades de tratamento antiviral para os pacientes com HC e o número médio de dias em tratamento para cada terapia.

O número médio anual de consultas para pacientes com tratamento antiviral indicadas por hepatologistas, gastroenterologistas e infectologistas é de 6,3 vezes ao ano, enquanto para pacientes sem tratamento antiviral é de duas vezes. Exames hematológicos são solicitados para 95,5\% dos casos, na freqüência de cinco a seis vezes por ano, e exames bioquímicos são realizados em todos os casos. Neste estágio da doença, não ocorre internação hospitalar. Na vigência de tratamento antiviral, procedimentos diagnósticos são realizados com maior freqüência.

O gasto total do acompanhamento clínico estimado por paciente/ano do grupo tratado com antiviral foi de $R \$ 2.007,12$ e, no grupo não tratado, de $\mathrm{R} \$ 980,89$. Nestes gastos, estão incluídos os honorários médicos, exames laboratoriais e procedimentos diagnósticos realizados para o acompanhamento do paciente, sem incluir o gasto do medicamento antiviral.

\section{Cirrose compensada}

$\mathrm{Na}$ cirrose compensada (CC), 37\% não recebem tratamento antiviral. Quando recebem tratamento, a Lamivudina é o antiviral de escolha em $82 \%$ dos casos.

Considerando os casos que recebem algum tipo de tratamento, a freqüência de consulta aos hepatologistas, infectologistas ou gastroenterologistas, ocorre, em média, seis vezes no ano. Exames laboratoriais (hematológicos, bioquímicos e testes sorológicos) são solicitados de $83 \%$ a $96 \%$ dos casos. Ultra-sonografia é realizada em 96\% dos casos e endoscopia digestiva alta em $81 \%$.

No período de um ano, $27,3 \%$ dos casos em tratamento antiviral são internados em enfermaria geral, 9,2\% em unidade de terapia intensiva (UTI) e $45 \%$ em enfermaria especializada de hepatologia. Enquanto que 23,3\% dos casos sem tratamento antiviral são internados em enfermaria geral, $6 \%$ em unidade de terapia intensiva (UTI) e 26,5\% em enfermaria especializada de hepatologia. 


\section{Tabela I - Gastos anuais estimados relativos ao acompanhamento clínico por paciente com cirrose compensada, com e sem tratamento antiviral}

\begin{tabular}{|c|c|c|}
\hline Tipo de gasto & om tratamento antiviral & Sem tratamento antiviral \\
\hline Honorários médicos & $R \$ 69,28$ & $R \$ 25,15$ \\
\hline Exames laboratoriais* & $R \$ 1.3012,07$ & $\mathrm{R} \$ 344,42$ \\
\hline Procedimentos diagnósticos $*$ ** & $R \$ 858,78$ & $R \$ 824,30$ \\
\hline Internação hospitalar & $\mathrm{R} \$ 44,60$ & $R \$ 31,30$ \\
\hline Total & $\mathrm{R} \$ 2.284,73$ & $\mathrm{R} \$ 1.243,17$ \\
\hline
\end{tabular}

* Exames laboratoriais: exames hematológicos, bioquímicosetestes sorológicos.

*** Procedimentos diagnósticos: biópsia hepática, ultra-sonografia hepática, tomografia computadorizadade abdome, ressonânciamagnética de abdome eendoscopia digestivaalta.

Os gastos anuais estimados relativos ao acompanhamento clínico por paciente com cirrose compensada, com e sem tratamento antiviral, estão listados na Tabela I.

\section{Cirrose descompensada}

Casos de cirrose descompensada (CD) necessitam freqüentemente de hospitalização. $O$ painel de especialista estimou que $59 \%$ destes pacientes são internados, em média, duas vezes durante o primeiro ano após a descompensação, com tempo médio de permanência de II dias em enfermaria.

Procedimentos diagnósticos e terapêuticos são realizados com maior freqüência neste grupo. Ultra-sonografia e endoscopia digestiva alta são realizadas de duas a três vezes ao ano.

O componente de maior gasto no tratamento das complicações destes casos foi o item medicamento, representando $\mathrm{R} \$$ 20. 184,50 por paciente/ano. $O$ gasto total estimado por paciente/ ano foi de $R \$ 22.022,61$. A Tabela 2 apresenta os gastos atribuídos aos pacientes com CD. Os medicamentos citados no quadro abaixo não dizem respeito a antivirais para o tratamento da Hepatite, e sim aos medicamentos usados para complicações inerentes à progressão da doença.

\section{Carcinoma hepatocelular}

Segundo o painel de especialistas, a estimativa é que casos de carcinoma hepatocelular (CHC) tenham, em média, sete consultas com hepatologistas no período de um ano. O hepatologista é o especialista responsável pelo tratamento do paciente com $\mathrm{CHC}$ em $81 \%$ dos casos. $O$ restante dos casos é atendido por gastroenterologista e oncologista. A razão do menor número de pacientes atendidos por oncologista nesta fase da doença é atribuída aos resultados insatisfatórios alcançados com quimioterapia e radioterapia. Os exames laboratoriais hematológicos e bioquímicos são solicitados com freqüência de sete vezes ao ano, por paciente. Procedimentos terapêuticos, tais como paracentese, quimioembolização e alcoolização são realizadas cerca de três
Tabela 2 - Gastos anuais estimados relativos aos pacientes com cirrose descompensada

\begin{tabular}{|c|c|}
\hline Tipo de gasto & Gasto anual por paciente \\
\hline Honorários médicos & $R \$ 105,66$ \\
\hline Exames laboratoriais* & $\mathrm{R} \$ 403,47$ \\
\hline Procedimentos diagnósticos e terapêuticos $*$ ** & $R \$ 1.116,15$ \\
\hline Internação hospitalar & $R \$ 212,83$ \\
\hline Medicamentos ${ }^{*} * * *$ & $R \$ 20.184,50$ \\
\hline Total & $\mathrm{R} \$ 22.022,61$ \\
\hline \multicolumn{2}{|c|}{$\begin{array}{l}\text { * Exames laboratoriais: exames hematológicos, bioquímicos etestes sorológicos. } \\
\text { *** Procedimentos diagnósticos eterapêuticos: biópsia hepática, ultra-sonografia hepática, tomografia } \\
\text { computadorizada de abdome, ressonância magnética de abdome, endoscopia digestivaalta, paracentese } \\
\text { diagnóstica, shuntcirúrgico, escleroterapia e paracenteseterapêutica. } \\
\text { ***** Medicamentos: relacionadosa ascite, relacionadosà hemorragia de varizes esofagianas, relacionadosà } \\
\text { encefalopatia hepática, antibióticos para peritonite bacteriana e suplementos nutricionais. }\end{array}$} \\
\hline
\end{tabular}

vezes ao ano por paciente. Segundo o painel de especialistas, pacientes com CHC apresentam alta probabilidade de internação hospitalar, com cerca de cinco hospitalizações/ano e tempo médio de permanência entre oito e doze dias, em enfermaria especializada.

$O$ presente estudo estima que 0 gasto anual por paciente com $\mathrm{CHC}$ devido à hepatite $\mathrm{B}$ fica em torno de $\mathrm{R} \$ 5$ mil. Estes gastos são referentes a consultas médicas, testes laboratoriais, procedimentos e hospitalizações, não considerando quimioterapia, citada anteriormente.

\section{Transplante hepático}

O gasto por paciente do transplante hepático no SUS, em 2004, foi de $R \$ 52.172,60$, e o gasto com administração da imunoglobulina da hepatite $B$, no pós-transplante, foi de $R \$$ $35.200,00$, totalizando gasto final de $R \$ 87.372,60$ no primeiro ano após realização do procedimento.

O gasto do procedimento com o transplante hepático contempla a retirada do fígado do doador e os gastos do implante hepático no paciente, tais como diária hospitalar, exames complementares, medicamentos, hemoterapia, taxa de uso do centro cirúrgico e material.

\section{Discussão}

Com base em estimativas produzidas por um painel de especialistas em hepatologia, infectologia e farmacoeconomia, a progressão da HCVB se associa à elevação do gasto médio por paciente, principalmente a partir do estágio de cirrose descompensada. Neste modelo de estimativa de padrão de condutas e de utilização de recursos, os pacientes submetidos a transplante hepático são os de maior gasto médio. 
Tabela 3 - Gasto anual de cinco estágios evolutivos da hepatite crônica B, em diferentes países, apresentados em ordem decrescente

\begin{tabular}{|c|c|c|c|c|c|c|}
\hline País - ano do estudo & Hepatite crônica & Cirrose compensada & Cirrose descompensada & Carcinoma hepatocelular & Transplante hepático & Total \\
\hline 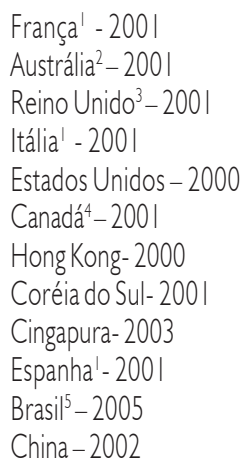 & $\begin{array}{l}\text { US\$1477 } \\
\text { US\$1019 } \\
\text { US\$3956 } \\
\text { US\$2487 } \\
\text { US\$761 } \\
\text { US\$1921 } \\
\text { US\$810 } \\
\text { US\$248 } \\
\text { US\$410 } \\
\text { US\$4589 } \\
\text { US\$ } 987 \\
\text { US\$142 }\end{array}$ & $\begin{array}{l}\text { US\$1532 } \\
\text { US\$1152 } \\
\text { US\$4416 } \\
\text { US\$2902 } \\
\text { US\$227 } \\
\text { US\$1921 } \\
\text { US\$1321 } \\
\text { US\$679 } \\
\text { US\$671 } \\
\text { US\$5401 } \\
\text { US\$1411 } \\
\text { US\$185 }\end{array}$ & $\begin{array}{l}\text { US\$11948 } \\
\text { US\$9885 } \\
\text { US\$17642 } \\
\text { US\$9813 } \\
\text { US\$11459 } \\
\text { US\$3405 } \\
\text { US\$7490 } \\
\text { US\$1419 } \\
\text { US\$8794 } \\
\text { US\$7151 } \\
\text { US\$8809 } \\
\text { US\$1701 }\end{array}$ & $\begin{array}{l}\text { US\$12637 } \\
\text { US\$9713 } \\
\text { US\$18624 } \\
\text { US\$9424 } \\
\text { US\$7533 } \\
\text { US\$9849 } \\
\text { US\$15618 } \\
\text { US\$3044 } \\
\text { US\$7036 } \\
\text { US\$5041 } \\
\text { US\$1905 } \\
\text { US\$4740 }\end{array}$ & $\begin{array}{l}\text { US\$1 I } 4281 \\
\text { US\$1 } 19332 \\
\text { US\$94306 } \\
\text { US\$88535 } \\
\text { US\$86552 } \\
\text { US\$86900 } \\
\text { US\$65961 } \\
\text { US\$67|56 } \\
\text { US\$49353 } \\
\text { US\$34006 } \\
\text { US\$34948 }\end{array}$ & $\begin{array}{l}\text { US\$141875 } \\
\text { US\$141101 } \\
\text { US\$138944 } \\
\text { US\$113161 } \\
\text { US\$106532 } \\
\text { US\$103996 } \\
\text { US\$91200 } \\
\text { US\$72546 } \\
\text { US\$66264 } \\
\text { US\$56188 } \\
\text { US\$48060 } \\
\text { US\$6768 }\end{array}$ \\
\hline
\end{tabular}

'Câmbio: US $\$ 1=\cdot 0,74 ;{ }^{2}$ Câmbio: US $\$ 1=$ AUD $\$ 1,21 ;{ }^{3}$ Câmbio: US $\$ 1=£ 0,50 ;{ }^{4}$ Câmbio: US $\$ 1=$ CAD $\$ 1, \mid 4 ;{ }^{5}$ Câmbio: US $\$ 1=\operatorname{R} \$ 2,50$

O gasto médio anual por paciente variou de $R \$ 462,37$ na hepatite crônica sem tratamento antiviral, até $R$ \$ 87.372,60 no primeiro ano após o transplante hepático. Os maiores determinantes de gasto, isto é, os componentes que causaram maior impacto econômico, foram os medicamentos e procedimentos terapêuticos.

A ascensão do gasto, nos diferentes estágios evolutivos, encontrado em nossos resultados assemelha-se aos estudos publicados sobre o custo em diferentes países do continente asiático, europeu e americano, com diferenças significativas nos valores absolutos encontrados (Tabela 3).

Apesar de se tratar de diferentes modelos de sistemas de saúde pesquisados e diferentes formas de coleta de dados, os componentes de maior custo são os medicamentos e as hospitalizações para realização de procedimentos nos estágios mais avançados da doença.

Os gastos associados ao tratamento da HCVB em Hong Kong e Cingapura, foram estimados na perspectiva do sistema público de saúde, valores ajustados para os anos 2000 e 2003, respectivamente $^{7}$. Hong Kong e Cingapura são regiões endêmicas para 0 HBV, com soroprevalência de $10 \%$ e $5 \%$, respectivamente. Os autores realizaram, retrospectivamente, pesquisa de dados primários em prontuários médicos e dividiram a doença em cinco estágios evolutivos. O gasto por paciente aumentou com a gravidade da doença e representou cerca de $4 \%$ dos gastos com saúde nestes países. A Tabela 3 resume os resultados.

$\mathrm{Na}$ Coréia do Sul, os gastos da HCVB e suas complicações foram estimados para 0 ano de $200 l^{8}$. Os autores pesquisaram a base de dados do Seguro Nacional de Saúde, registros de pacientes e opinião de especialistas. O principal componente de custo foi a hospitalização, incluindo procedimentos cirúrgicos (Tabela 3).

Em amostra de pacientes de Beijing, foi identificada elevação nos gastos dos quatro estágios evolutivos da HCVB, em análise retrospectiva de prontuários médicos, valores ajustados para 0 ano de $2002^{9}$. Os autores estimaram variação do gasto médio por paciente, de US\$ I 42 na hepatite crônica sem cirrose, até US\$ 4.74I no
CHC, em um ano (Tabela 3).

Os gastos na França, Itália, Espanha e Reino Unido, decorrentes do tratamento da HCVB e suas complicações, foram estimados na perspectiva do sistema público de saúde, valores ajustados para o ano de $200 \mathrm{I}^{10}$. Foi aplicado questionário para coleta de dados com especialistas, para documentar o padrão de tratamento da HCVB nestes países e os recursos utilizados (Tabela 3).

Nos Estados Unidos", foram analisados os gastos associados à infecção crônica pelo vírus $\mathrm{B}$, por meio de dados retrospectivos de faturamento das empresas de cuidados gerenciados de saúde, valores ajustados para o ano 2000.

O gasto com medicações foi o principal componente nos estágios de hepatite crônica e cirrose compensada, enquanto hospitalização representou o maior gasto nos outros estágios evolutivos (Tabela 3).

Por meio de painel com oito especialistas e análise de prontuários médicos, avaliou-se o padrão de tratamento da hepatite crônica B em nove províncias canadenses, para estimava de gasto, valores em dólar canadense, ajustados para 0 ano de $200 \mathrm{I}^{12}$. 0 gasto médio por paciente variou de US\$2.191 na hepatite crônica, até US\$99.066 no transplante hepático, em um ano. Os maiores componentes de custo foram medicações e hospitalizações (Tabela 3).

Na Austrália, o gasto com o tratamento da hepatite crônica B foi estimado, valores em dólar australiano, ajustados para $\mathrm{O}$ ano de $200 I^{13}$. Os autores realizaram análise estatística multivariada e verificaram que idade, status conjugal, país de origem e duração do seguimento não foram estatisticamente significativos para explicar a variação de gastos (Tabela 3).

Os pacientes infectados pelo VHB, submetidos a transplante hepático e que não fazem profilaxia adequada, apresentam recorrência da infecção no enxerto em mais de $80 \%$ dos casos, o que determina insucesso em muitos pacientes. A sobrevida após o transplante hepático dos pacientes AgHBs positivos é significativamente menor quando comparados com os AgHBs negativos, dependendo, entre outras variáveis, do status sorológico e 
virológico antes do transplante. A administração de imunoglobulina da hepatite $B$, durante e depois do transplante, previne a recorrência da hepatite no enxerto em significativa proporção de pacientes infectados pelo HBV. Em estudo multicêntrico europeu, a taxa de recorrência foi de $75 \%$ em pacientes que não usaram imunoglobulina hiperimune para hepatite $B$, ou a receberam por curto período, e de $33 \%$ naqueles que a receberam por longo tempo. A administração desta imunoglobulina, em longo prazo, reduziu a taxa de recorrência a menos de $10 \%$ nos pacientes com hepatite fulminante, e a menos de $30 \%$ naqueles com cirrose HBVDNA negativos, mas não diminuiu a taxa de recorrência nos pacientes cirróticos HBV-DNA positivos. Porém, mesmo nestes doentes, é possível evitar a reinfecção do enxerto, desde que sejam utilizadas doses maiores de imunoglobulina. A necessidade de administração da imunoglobulina no pós-transplante é o principal componente de gasto destes pacientes.

Estudos recentes ${ }^{14,15}$ sugeriram que os riscos para desenvolvimento de cirrose descompensada e carcinoma hepatocelular são maiores em pacientes com níveis de carga viral persistentemente $>10^{5}$ cópias $/ \mathrm{mL}$, e que tratamentos capazes de manter cargas virais abaixo destes níveis retardam ou previnem estas complicações. Dados de um recente e amplo estudo epidemiológico relacionando carga viral e carcinoma hepatocelular ${ }^{14}$ relatam que aumentos de carga viral de 10.000-99.999 para 100.000-999.999 cópias/mL aumentam as taxas de incidência de carcinoma hepatocelular de 297/100.000 paciente-anos para 962/100.000 paciente-anos. Similarmente, de acordo com lloeje et al ${ }^{15}$, elevações da carga viral de 10.000-99.999 para 100.000-999.999 cópias/mL podem aumentar as taxas de incidência de cirrose de 774/100.000 paciente-anos para 1.878/100.000 paciente-anos.

Os achados deste estudo devem ser interpretados com cautela, dado algumas limitações da metodologia utilizada. $\mathrm{Na}$ realidade, a análise baseou-se na premissa de que os pacientes com HCVB tratados na rede do SUS recebem o tratamento preconizado por um painel de especialistas. Há razões para acreditar que esta não é a realidade do sistema público de saúde no país. Por conseguinte, a coleta de dados primários em hospitais da rede SUS, apesar de traduzir mais fielmente a realidade destes centros, avaliaria padrões de condutas diagnósticas e terapêuticas provavelmente subótimas. O atendimento oferecido no SUS é influenciado, ou quiçá ditado, pelo reembolso fixo praticado de acordo com o diagnóstico, e não com o efetivamente consumido no tratamento de determinado paciente. Assim sendo, coleta efetiva, não estimada, de dados primários na rede hospitalar também tem suas limitações.

Outra limitação foi a ausência de estimativa dos custos indiretos, resultantes da perda de produtividade associada ao absenteísmo e à mortalidade precoce decorrente da HCVB. O custo indireto representa importante ônus para a sociedade e deve ser contemplado pelos formuladores de políticas de saúde.

\section{ConClusão}

Em suma, a progressão da HCVB se associa com a elevação do gasto médio por paciente, principalmente a partir do estágio de
$C D$, condição clínica que exige várias intervenções terapêuticas onerosas para o Sistema de Saúde. Os procedimentos e medicamentos representam os principais componentes do gasto com a HCVB.

Conflito de interesse: Este estudo foi patrocinado pela Bristol Myers Squibb Farmacêutica Ltda. Dr. Adauto Castelo Filho participa de protocolos de investigaçãa clínica e atua como consultor científico para Boheringer-Ingelheim e Bristol-Meyers Squibb e realiza palestras para Bristol-Myers Squibb. Dr. Mario Guimarães Pessoa - participa de protocolos de investigação clínica e consultor científico para Bristol-Myers Squibb, Human Genome Sciences, Glaxo Smith Kline, Novartis e Roche. Dr. Denizar Vianna - Consultor científico para AstraZeneca, Abbott, Bristol-Myers Squibb, MS, Boehringer, Eli Lilly, Genzyme, GlaxoSmithKline, Janssen Cillag, Roche, Roche Diagnostic, Mantecorp, Merck, Novartis, Novartis OTC, Pfizer, Sanofi-Aventis, Serono e Support. Dra. Tânia Barreto - Gerente de Departamento Médico da BristolMyers Squibb. Marcia Alves - Coordenadora de Farmacoeconomia da Bristol-Myers Squibb.

\section{SUMMARY}

COST ESTIMATES OF CHRONIC HEPATITIS B VIRUS FOR THE BRAZILIAN UNIFIED HEALTH SYSTEM IN 2005

BaCKGround. Chronic Hepatitis B Virus (CHBV) is a disease that places a large financial burden on healthcare systems and society.

OBJECTIVE. The aim of this study was to estimate patient management patterns, and associated medical resource utilization and expenses, for each of the four stages of chronic HBV infection in the public unified healthcare system settings, in 2005.

METHODS. An expert panel comprised of ten physicians, leading specialists in hepathology and infectious diseases, was convened to obtain information regarding management of CHBV patients in Brazil. Expense inputs were mainly obtained from government fee schedules and pharmaceutical price tables. Costs were estimated under the perspective of the public health system. Data were analyzed using Windows SPSS version 12.0.

RESULTS. Estimated patient expenses were calculated for the four stages of CHBV infection. The estimated annual expenses per patient were: $R \$ 980.89$ (US\$392) for chronic hepatitis B with no cirrhosis and without antiviral therapy; $R \$ 1,243.17$ (US\$ 496) for compensated cirrhosis without antiviral therapy; $R \$ 22,022.61$ (US\$ 8809) for decompensated cirrhosis; $R \$ 4,764.95$ (US\$ 1,905) for hepatocellular carcinoma; and $R \$ 87,372.60$ (US\$ 34,948) for liver transplant.

CONCLUSION. Estimated expenses associated with drugs and procedures represented the main components of the expenses of CHBV infection. In this model, expenses increase dramatically as the disease progresses to more advanced stages, suggesting that over the long term delaying progression may reduce costs. [Rev Assoc Med Bras 2007; 53(6): 486-9I]

KEY words: Hepatitis B. Chronic. Costs and cost analysis. Health care costs. 


\section{REFERÊNCIAS}

I. Chang MH, Chen C], Lai MS, Hsu HM, Wu TC, Kong MS, et al. Universal hepatitis B vaccination in Taiwan and incidence of hepatocellular carcinoma in children: Taiwan Childhood Hepatoma Study Group. N Engl J Med. 1997:336:1855-9.

2. Silveira TR, Fonseca JC, Rivera L, Fay OH, Tapia R, Santos JI, et al. La seroprevalencia de hepatitis B en América Latina. Rev Panam Salud Publica. 1999;6:378-83.

3. Bensabath G, Hadler SC, Soares MC, Fields H, Dias LB, Popper H, et al. Hepatitis dela virus infection and Labrea hepatitis in the Amazon basin. JAMA. 1987;258:479-83.

4. Focaccia R, Conceição OJG, Junior HS, Sabino E, Bassit L, Nitrini DR, et al. Estimated prevalence of viral hepatitis in the general population of the municipality of São Paulo, measured by a serologic survey of a stratified, randomized and residence-based population. Braz J Infect Dis. 1998:2:269-84.

5. Food and Drug Administration. U.S. Department of Health and Human Services. Rockville; 2005. [cited 2006 jul 17]. Available from: http:// www.fda.gov.

6. DATASUS. Ministério da Saúde. Brasília; 2005. [cited 2006 jul 17]. Disponível em: http://www.datasus.gov.br.

7. Li SC, Ong SC, Lim SG, Yeoh KG, Kwong KS, Lee V, et al. A cost comparison of management of chronic hepatitis $b$ and its associated complications in Hong Kong and Singapore. J Clin Gastroenterol. 2004; 38(Suppl 10) SI36-43.

8. Yang BM, Kim CH, Kim JY. Cost of chronic hepatitis B infection in South Korea. J Clin Gastroenterol. 2004; 38(Suppl 10):SI53-7.
9. Zhigiang G, Zhaohui D, Qinhuan W, Dexian C, Yunyun F, Hongtao L, et al. Cost of chronic hepatitis in China. J Clin Gastroenterol. 2004;38(I OSuppl):SI75-8.

10. Brown RE, De Cock ED, Colin X, Antonanzas F, lloeje UH. Hepatitis B management cost in France, Italy, Spain and the United Kingdom. J Clin Gastroenterol. 2004;38(Suppl 10):S169-74.

II. Lee TA, Veenstra DL, Iloeje UH, Sullivan SD. Costs of chronic hepatitis B infection in the United States. J Clin Gastroenterol. 2004;38(Suppl 10):SI 44-7.

12. Gagnon YM, Levy AR, lloeje UH, Briggs AH. Treatment costs in Canada of health conditions resulting from chronic hepatitis B infection. J Clin Gastroenterol. 2004:38(Suppl I0):SI79-86.

13. Butler JR, Pianko S, Korda RJ, Nquyen S, Gow PJ, Roberts SK, et al. The direct cost of managing patients with chronic hepatitis B infection in Australia. J Clin Gastroenterol. 2004;38(SuppI 10):SI87-92.

14. Chen J $C$ et al. Risk of hepatocellular carcinoma across biological gradient of serum hepatits B virus DNA level. JAMA. 2006;295:65-73.

I 5. Iloeje UH, Yang HI, Su J, Jen CL, You SL, Chen CJ, et al. Predicting cirrosis risk based on the level of circulating hepatitis B viral Load. Gastroenterology. 2006;130:678-86.

Artigo recebido: 27/10/06

Aceito para publicação: 21/08/07 